\title{
Improving Future High-Energy Tau Neutrino Searches in IceCube
}

\section{The IceCube Collaboration ${ }^{\dagger}$}

$\dagger$ http://icecube.wisc.edu/collaboration/authors/icrc17_icecube

E-mail: juliana.stachurska@icecube.wisc.edu

\begin{abstract}
One of the prime goals of the IceCube Neutrino Observatory is to identify tau neutrinos in the astrophysical neutrino flux. The most recent tau neutrino search is based on the high-energy starting event (HESE) sample and has not found any events that could be classified as tau neutrinos. However, this sample rejects all events in the outer detector region to suppress atmospheric background. Given the low expected number of identifiable tau neutrino events it is desirable to combine different suitable event selections in future searches. Here we present an approach where we use an event selection based on a shower-like event signature rather than a fiducial volume veto. This retains events in the outer parts of the detector while greatly reducing background from track-like events. We identify tau neutrino events by means of direct reconstruction of the double cascade topology. Based on Monte Carlo studies, combining this sample and the HESE sample will enhance the number of identifiable tau neutrino events by $\sim 20-45 \%$ for a given observation time at a similar background level.
\end{abstract}

Corresponding authors: J.Stachurska, M. Usner*

DESY, Platanenallee 6, D-15738 Zeuthen, Germany

35th International Cosmic Ray Conference - ICRC2017

10-20 July, 2017

Bexco, Busan, Korea

${ }^{*}$ Speaker. 


\section{Introduction}

The IceCube Neutrino Observatory is a $\mathrm{km}^{3}$ Cherenkov detector at the South Pole [1]. It discovered an astrophysical flux of high energy neutrinos in 2012 [2]. The highest energies deposited in the detector by neutrino interactions are well above $1 \mathrm{PeV}$, meaning that the neutrinos responsible cannot have been produced locally in the Earth's atmosphere and must be of cosmic origin. If the dominant production mechanism is $\pi^{ \pm}$decay resulting in a flavor ratio of $v_{e}: v_{\mu}: v_{\tau}$ of $1: 2: 0$ at the source, the expected flavor ratio on Earth is $v_{e}: v_{\mu}: v_{\tau} \approx 1: 1: 1$ due to neutrino mixing [3]. Therefore, we expect one third of the astrophysical flux measured with IceCube to be caused by $v_{\tau}$ interactions. With a negligible atmospheric $v_{\tau}$ content $[4,5]$, identifying $v_{\tau}$ interactions in the IceCube data would be yet another smoking gun signature of the cosmic origin of the highest-energy neutrino flux. However, to this day no $v_{\tau}$ event has been identified with IceCube [6, 7].

There are three event topologies in IceCube to be distinguished. Track-like events stem from charged current $v_{\mu}$ interactions and atmospheric muons, as well as charged current $v_{\tau}$ interactions where the $\tau$ lepton decays muonically (branching ratio of $\sim 17 \%$ ). Cascade-like events come from $v_{e}$ or neutral current interactions, and from charged current $v_{\tau}$ interactions where the $\tau$ lepton decays too quickly to be resolved.

Only charged current $v_{\tau}$ interactions can create a double cascade [8], where the first cascade comes from the neutrino interaction and $\tau$ production, and the second from the $\tau$ decaying electromagnetically or hadronically (branching ratio of $\sim 83 \%$ ). However, the $\tau$ lepton has a mean decay length of $\left\langle L_{\tau}\right\rangle \sim 50 \mathrm{~m} \cdot E_{\tau}[\mathrm{PeV}]$, where $E_{\tau}$ is the $\tau$ energy. Therefore, charged current $v_{\tau}$ interactions become distinguishable from $v_{e}$ or neutral current interactions only at energies $\gtrsim 100 \mathrm{TeV}$. More details on these event topologies can be found in [7]. So far, two dedicated $v_{\tau}$ analyses have been presented using different event selections. While [6] looked for double pulse signatures in the waveform of individual digital optical modules (DOMs) in 3 years of IceCube data, [7] searched for double cascades using direct reconstruction in 6 years of IceCube data. At neutrino energies $\gtrsim 100 \mathrm{TeV}$, where the $\tau$ lepton can live long enough to create a resolvable double cascade signature in the detector, the measured neutrino flux is rather low. Here we present an approach to increase the event sample used for the double cascade search by combining different selections of high-energy neutrino events.

\section{Data Samples}

\subsection{High-Energy Starting Events (HESE)}

The HESE sample is based on the event selection described in [2]. A veto layer is used to suppress incoming atmospheric background, resulting in a decreased effective volume. All events are thereby required to start inside the detector. It is an all-flavor event selection, with events classified as cascade-like or track-like. As the HESE event selection does not discriminate event shapes, it retains a similarly high efficiency for $v_{\tau}$ events at all tau decay lengths.

In the years 2012-2016, four cascade-like events with energies above $100 \mathrm{TeV}$ were seen in HESE, but not in the cascade sample described below, as they did not fulfill all of the cascadeselection criteria. The currently available HESE sample contains 49 events with a deposited energy above $60 \mathrm{TeV}$ in the years 2010-2016, with an estimated background of 9 atmospheric neutrino 
events and 2 atmospheric muons [9]. This is the event selection used in [7], where the third eventtopology of a double cascade has been introduced.

\subsection{Contained Cascade Events}

The contained cascade event selection makes use of almost the entire instrumented volume and uses topological criteria like the sphericity of the Cherenkov light pattern to select cascade-like events and reject track-like events $[10,11]$. Therefore, the efficiency of the cascade event selection to $v_{\tau}$ events is highest for events with short tau decay lengths that have spherical light patterns and decreases with increasing tau decay lengths.

The sample consists of six years (2010-2016) of cascades, of which the high- and mediumenergy part above $\sim 10 \mathrm{TeV}$ deposited energy will be used. In the time period between 2012 and 2016 there are 19 events with energies above $100 \mathrm{TeV}$, ten of which are not in the HESE sample. This includes one event originating from an interaction in a layer of ice where light is strongly attenuated due to a high concentration of dust particles. There is no contamination from atmospheric muons expected above $60 \mathrm{TeV}$ deposited energy.

Due to the steeply falling spectrum of the astrophysical neutrino flux, most of the identifiable double cascade signal is expected to have tau decay lengths close to the resolution threshold. Therefore, the high-energy part of the cascade sample provides an excellent addition for a search for $v_{\tau}$ interactions. The work presented here is the first study aimed at identifying $v_{\tau}$ events in the contained cascade sample.

\subsection{Uncontained Cascade Events}

The outer parts of the detector are used as a veto region to varying degrees in both event selections described above. As shown in [10,12], this area of the detector allows a reasonably good separation between astrophysical neutrino signal and atmospheric muon background. Events in the uncontained cascade sample have interaction vertices reconstructed at the rim of the detector, either close to the outer strings or below the instrumented volume. To suppress background from atmospheric muons, events with vertices at the corners of the detector are discarded and only highenergy events are kept. The event selection is designed to provide a sample that is statistically independent from the contained cascades sample. So far, it has been applied to two years (20102011) of data, but efforts are underway to extend the event selection to all currently available data. While this sample offers a gain in the number of identifiable $v_{\tau}$ events, it is more difficult to reconstruct events not contained inside the detector with the presently used algorithms. Therefore, the addition of the uncontained cascade event sample for a $v_{\tau}$ search is desirable, but challenging. This sample has not yet been used for follow-up $v_{\tau}$ searches, either.

The event numbers for the published samples are summarized in Table 1, showing the number of events with reconstructed energies $>100 \mathrm{TeV}$ for all topologies (cascades and tracks), cascades only and cascades found only in that event selection. The effective areas for tau neutrinos for the three described event selections are shown in Figure 1. Note that the uncontained cascade event selection has only been applied to two years of data, and efforts are presently ongoing to extend the event selection; its effective area might therefore change. 


\begin{tabular}{c|c|c|c} 
event numbers $(>100 \mathrm{TeV})$ & all topologies & cascades & unique cascades \\
\hline HESE 6-year & 27 & 22 & 7 \\
contained cascades 6-year & 26 & 26 & 14 \\
uncontained cascades 2-year & 9 & 9 & 8
\end{tabular}

Table 1: Number of events with $>100 \mathrm{TeV}$ reconstructed energy observed in the three samples considered. The number of events for all topologies, cascades, and the number of cascades found only in the given sample are shown. Note that the published uncontained cascade sample covers two years only.

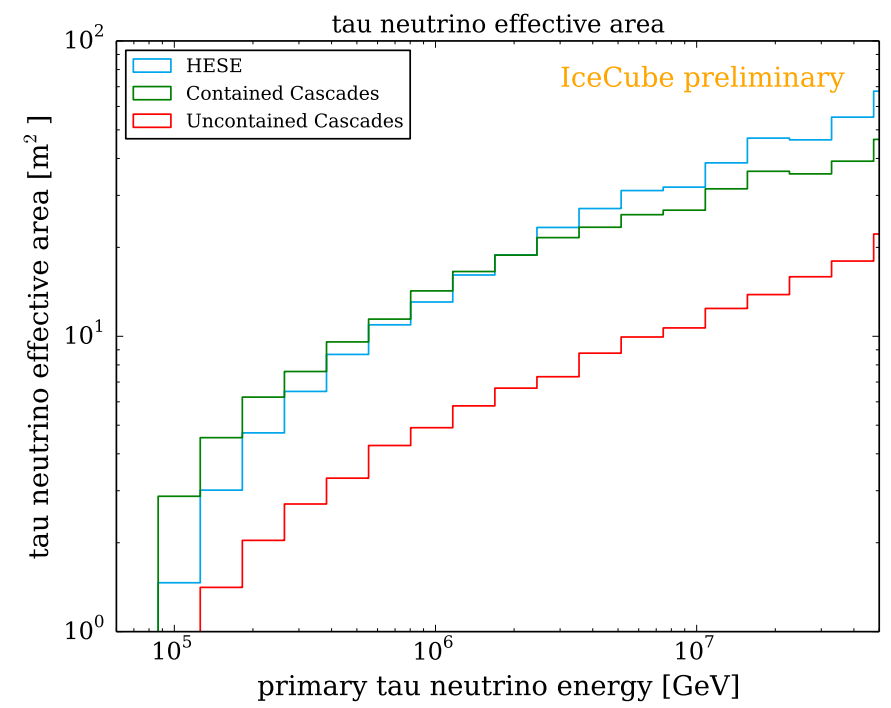

Figure 1: Effective areas for tau neutrino interactions using the HESE, contained cascade, and uncontained cascade event selections. Note that the uncontained cascade event selection is currently studied and might change.

\section{Observables sensitive for tau neutrino identification}

This study is done using a reconstruction where an algorithm maximizes the likelihood of the given event's light deposition using the double cascade hypothesis [7, 13]. Out of the nine fit parameters, three are used to identify double cascades: the energy of the first (hadronic) cascade creating a $\tau$ lepton, the length the $\tau$ lepton travels before it decays, and the energy of the second (hadronic or electromagnetic) cascade of the $\tau$ decay. Figure 2 shows the simulated survival rate of astrophysical tau neutrinos with deposited energies above $60 \mathrm{TeV}$ producing a double cascade as a function of tau decay length in the HESE, combined HESE + contained cascades, and combined HESE + contained cascades + uncontained cascades samples. Assuming an astrophysical flux of $\Phi_{v_{\tau}}=1.0 \cdot 10^{-18}\left(\frac{E}{100 \mathrm{TeV}}\right)^{-2} \mathrm{GeV}^{-1} \mathrm{~cm}^{-2} \mathrm{~s}^{-1} \mathrm{sr}^{-1}, 3.65$ tau neutrino double cascade events per year are expected in the combined sample, but only $1.36(0.86)$ of these have a resolvable tau decay length of $>10 \mathrm{~m}(>20 \mathrm{~m})$. The gain from including the contained cascade sample reaches $\sim 50 \%$ for the lowest resolvable lengths of $\sim 10 \mathrm{~m}$ as compared to the HESE sample alone, and $\sim 80 \%$ if both contained and uncontained cascades are included. The challenge is to resolve as many of the events with short decay lengths as possible. 


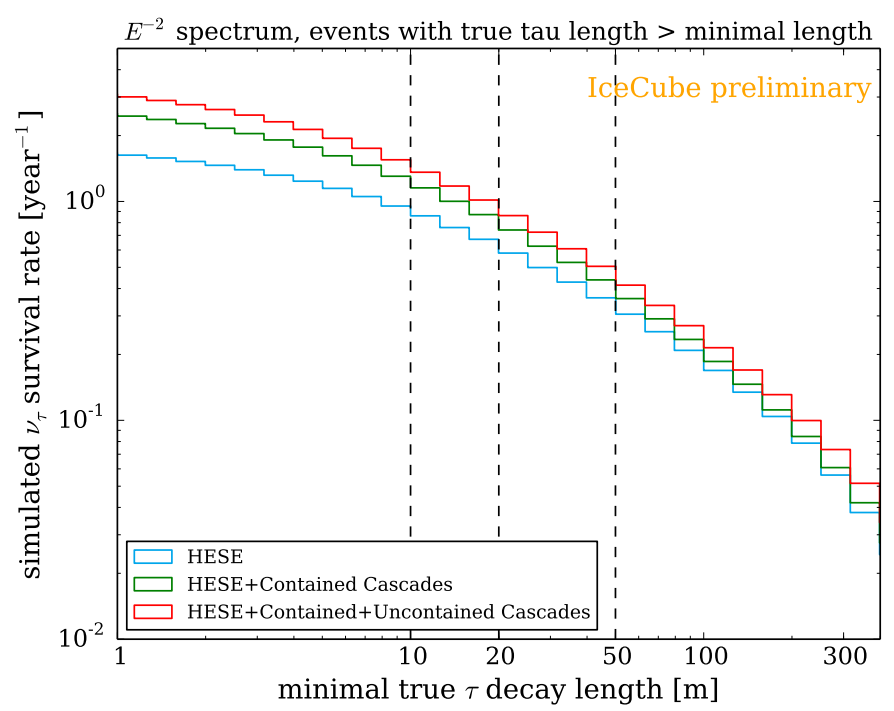

Figure 2: Simulated survival rates of astrophysical tau neutrino interactions with deposited energies above $60 \mathrm{TeV}$ producing a double cascade as a function of tau decay length in the HESE, combined HESE + contained cascades, and combined HESE + contained cascades + uncontained cascades samples, assuming an astrophysical flux of $\Phi_{v_{\tau}}=1.0 \cdot 10^{-18}\left(\frac{E}{100 \mathrm{TeV}}\right)^{-2} \mathrm{GeV}^{-1} \mathrm{~cm}^{-2} \mathrm{~s}^{-1} \mathrm{sr}^{-1}$.

The main goal of this study is then separating signal from background events based on the observables of the fit and classifying them as either double cascade, single cascade, or track in order to obtain signal and background rates for the combined data sample. Please note that the separation criteria here are not identical to the ones used in [7], and that we introduce a new variable in this work, the likelihood ratio, which we describe below. Only charged current $v_{\tau}$ interactions can create a true double cascade. However, the shorter the tau decay length is, the more does the light pattern resemble that of a single cascade. Large stochastic energy losses along a muon track can mimic a double cascade.

A useful observable is the tau decay length $L_{\tau}$, where the algorithm gives a good resolution above $10 \mathrm{~m}$. Single cascades tend to be reconstructed as either double cascades having small (below $10 \mathrm{~m}$ ) separation lengths, or with an arbitrary separation length and one of the cascades having almost no energy. The latter is easily explained by a random noise hit somewhere in the detector far away from the neutrino interaction. For its removal the energy asymmetry $A_{E}:=\frac{E_{1}-E_{2}}{E_{1}+E_{2}}$ is used, with $E_{1}\left(E_{2}\right)$ being the reconstructed energy of the first (second) cascade. For single cascades, the values for $A_{E}$ peak at \pm 1 . Thus, events with high positive or negative energy asymmetries are classified as single cascades. In this work, we introduce a new parameter to distinguish double cascades from single cascades. We fix the tau decay length to $0 \mathrm{~m}$ and use the reconstruction algorithm to fit both cascades at the same point, thus making it a single cascade. The likelihood values are compared to the free minimization, and the event is only classified as a double cascade if the test statistics $-2 \log \frac{L(0 \mathrm{~m})}{\mathcal{L} \text { (bestfit })}>1$ is fulfilled. This is a powerful tool to distinguish between single and double cascades, as quite naturally, the single cascade hypothesis describes a real single cascade better.

Looking at track-like background, the energy asymmetry does not have a strong separation 
power. There is, however, a physical correlation between the mean tau decay length and its energy: as mentioned above, $\left\langle L_{\tau}\right\rangle \sim 50 \mathrm{~m} \cdot E_{\tau}[\mathrm{PeV}]$, albeit with a large spread about the average value. This can be used to define a signal region in $L_{\tau}$-vs.- $E_{2}$ space, classifying events with $L_{\tau}<500 \mathrm{~m} \cdot E_{2}$ [PeV] as signal-like, and events with $L_{\tau} \geq 500 \mathrm{~m} \cdot E_{2}[\mathrm{PeV}]$ as track-like (this concept is illustrated in Figure 2 of [7]). These cuts allow for a $90 \%$ double cascade purity in the signal sample selected from contained cascades.

To get the same purity in the sample containing the additional HESE events, one more variable has to be used in addition to the cuts developed for the contained cascade sample as a final step to reduce the remaining track-like background. We use the energy confinement, i.e. the fraction of the total energy that is deposited in the vicinity of the reconstructed cascade vertices. Only events with a confined energy fraction close to 1.0 remain in the double cascade sample.

As the reconstruction algorithms used for this study have only been tested for events contained inside the detector volume, the study initially focused on double cascades with both vertices reconstructed inside the detector. This restriction poses a major challenge when aiming to extend the search for $v_{\tau}$ interaction to events that do not have both vertices contained inside the detector. Currently the performance of the algorithm and cuts for events that are outside the detector boundaries is being studied.

\section{Results from the combined sample}

The expected signal and background rates are investigated for the contained cascade and the HESE sample separately, as well as for the combination of both samples. Note that the contained cascade sample has significant overlap with the HESE selection; 50\% of the events in the combined sample pass both event selections. While there is no overlap between contained and uncontained cascades by construction, there is some overlap between the HESE and uncontained cascades
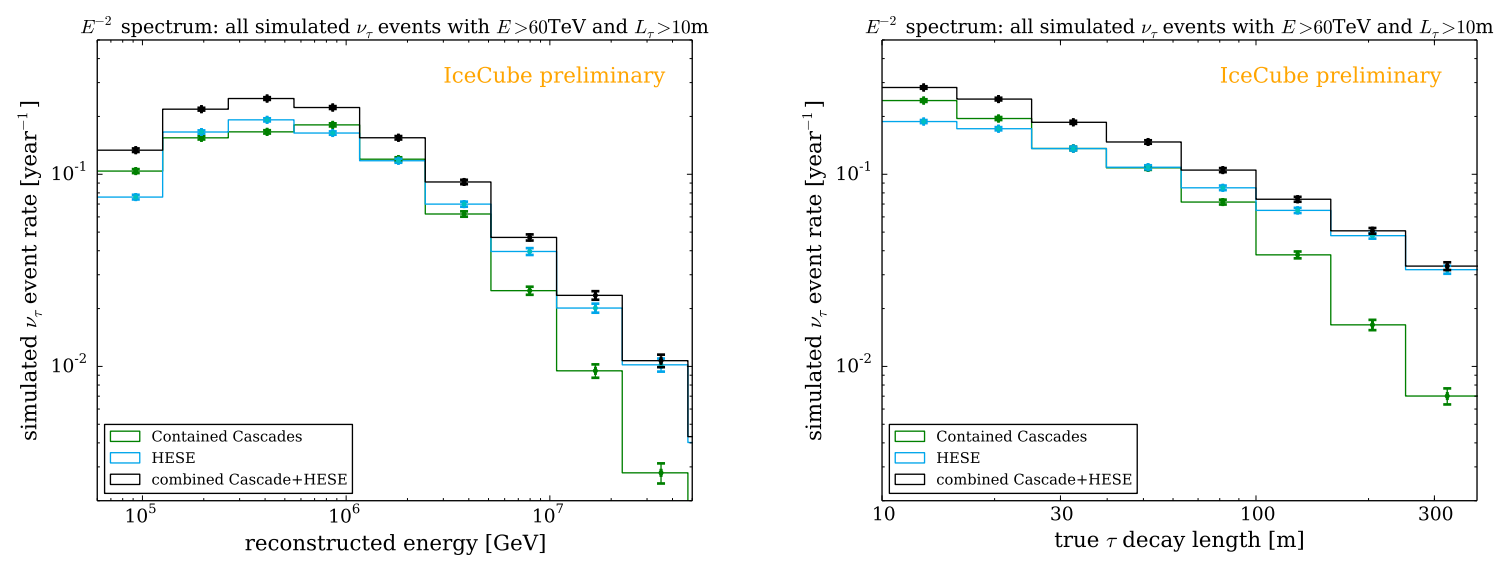

Figure 3: Simulated passing rates of astrophysical tau neutrino interactions with deposited energies above $60 \mathrm{TeV}$ producing a double cascade with separation $>10 \mathrm{~m}$ in the contained cascades and HESE event selection, and the combined sample for the same flux as in Figure 2. Left: Passing rate as a function of energy. Right: Passing rate as a function of tau decay length. 
samples from events at the bottom of the detector. This has been taken into account properly when combining the samples.

Figure 3 shows the passing rates of astrophysical tau neutrino interactions in the contained cascades and HESE samples, as well as in the combined sample. Given the nature of the event selections considered here, it is not surprising that the cascade sample retains many $v_{\tau}$ events with low tau decay lengths, while the HESE sample has a much higher efficiency for $v_{\tau}$ events with tau decay lengths above $\sim 100 \mathrm{~m}$, as the contained cascades event selection selects shower-like topologies and rejects events with elongated or track-like light patterns. Combining both samples for a search for $v_{\tau}$-interactions is thus desirable.

Our results are shown in Figure 4. For reconstructed tau decay lengths $>20 \mathrm{~m}$, the combined sample will lead to an increase in identifiable tau neutrino events by $\sim 30 \%$ as compared to the HESE selection alone assuming a hard spectrum with a spectral index of $\gamma=-2$. The expected gain in the $10-20 \mathrm{~m}$ separation length region is even higher at $\sim 50 \%$. With this spectrum, $\sim 3$ signal events with separation lengths above $20 \mathrm{~m}$ are expected in the combined sample with eight years of data, at a background level of $10 \%$. With a measured spectral index of $\gamma-2.48 \pm 0.08$ in the cascade sample [11], the number of very high-energy events is expected to be lower. Therefore, it is even more important to extend the $v_{\tau}$ search to the cascade sample, as it has a larger effective area than HESE at lower energies and separation lengths. For the softer spectral index of -2.48 , $\sim 2$ events are expected in the combined sample for eight years of data, for separation lengths $>20 \mathrm{~m}$. The gain from including the cascade sample is shown in Table 2 for both spectra.

The uncontained event selection provides additional events not seen in either of the other samples. However, the performance of the double cascade reconstruction algorithm on uncontained events needs to be studied further. Assuming that a similar efficiency as for contained events can

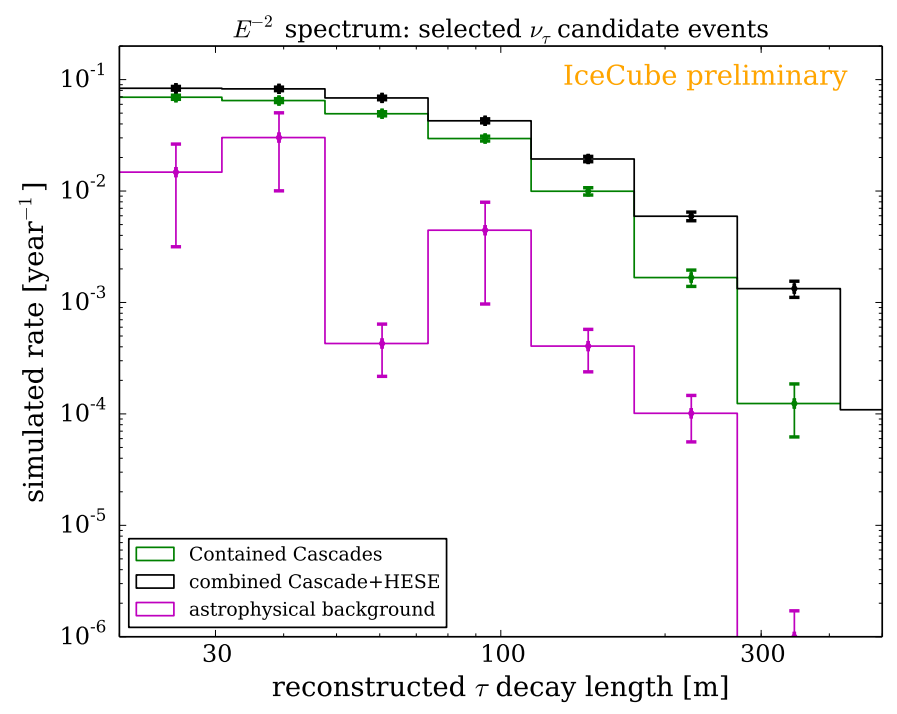

Figure 4: Simulated rates of identifiable astrophysical tau neutrino interactions with a reconstructed double cascade with separation $>20 \mathrm{~m}$ in the contained cascades and the contained cascades + HESE combined sample after cuts have been applied, assuming the same flux as in Figure 2. 


\begin{tabular}{c|c|c|c|c} 
energy spectrum & \multicolumn{2}{|c|}{$E^{-2}$} & \multicolumn{2}{c}{$E^{-2.48}$} \\
\hline tau decay length & $10-20 \mathrm{~m}$ & $>20 \mathrm{~m}$ & $10-20 \mathrm{~m}$ & $>20 \mathrm{~m}$ \\
\hline gain from including & $\sim 50 \%$ & $\sim 30 \%$ & $\sim 65 \%$ & $\sim 35 \%$ \\
cascades & $\sim 40 \%$ & \multicolumn{2}{|c}{$\sim 50 \%$}
\end{tabular}

Table 2: Gain in the observable number of events with $>60 \mathrm{TeV}$ deposited energy from combining the contained cascade sample with the HESE sample. The $E^{-2}$ spectral index denotes the flux used in Figure 2, the $E^{-2.48}$ spectral index denotes the flux observed in [11].

be achieved, an additional $\sim 15 \%$ identifiable tau neutrino events can be added to the combined HESE and contained cascades sample.

\section{Summary and Outlook}

Six years of IceCube data have not yet revealed astrophysical $v_{\tau}$ interactions. However, the most promising search to date has been based on only the HESE sample, with an expectation of $\sim 2$ identifiable $v_{\tau}$ events [7]. Combining this effort with the high-energy part of the contained cascade sample increases the expected number of identifiable $v_{\tau}$ events by $\sim 30 \%$ in the almost background free region of separation lengths $\geq 20 \mathrm{~m}$ and by $\sim 50 \%$ for separation lengths of $10-20 \mathrm{~m}$. For a softer spectrum, the importance of combining the two samples is even higher, and the gain from incorporating the cascade sample is $\sim 65 \%$ for separation lengths of $10-20 \mathrm{~m}$.

Another not yet incorporated sample is given by the uncontained cascades. While the reconstruction algorithms used have been developed for events that are contained within the fiducial volume, a study of their efficiency on not fully contained events is underway. If a good separation between double cascades and background can be achieved, another $\sim 15 \%$ gain in $v_{\tau}$ events (compared to the combined sample of contained cascades and HESE) is possible.

\section{References}

[1] IceCube Collaboration, M. G. Aartsen et al., JINST 12 (2017) P03012.

[2] IceCube Collaboration, M. G. Aartsen et al., Science 342 (2013).

[3] M. Bustamante, J. F. Beacom, and W. Winter, Phys. Rev. Lett. 115 (2015) 161302.

[4] M. Honda, T. Kajita, K. Kasahara, S. Midorikawa and T. Sanuki, Phys. Rev. D 75 (2007) 043006.

[5] R. Enberg, M. H. Reno, and I. Sarcevic, Phys. Rev. D 78 (2008) 043005.

[6] IceCube Collaboration, M. G. Aartsen et al., Phys. Rev. D93 (2016) 022001.

[7] IceCube Collaboration, PoS ( ICRC2017) 974 (these proceedings).

[8] J. G. Learned and S. Pakvasa, Astropart. Phys. 3 (1995) 267-274.

[9] IceCube Collaboration, PoS ( ICRC2017) 981 (these proceedings).

[10] IceCube Collaboration, PoS ( ICRC2015) 1109 (2016).

[11] IceCube Collaboration, POS ( ICRC2017) 968 (these proceedings).

[12] Stoessl, A., PhD thesis (2015).

[13] IceCube Collaboration, M. G. Aartsen et al., JINST 9 (2014) P03009. 